\title{
PREDICTING FLEXURAL STRENGTH OF CONCRETES INCORPORATING RIVER GRAVEL USING MULTI-LAYER PERCEPTRON NETWORKS: A CASE STUDY OF EASTERN NIGERIA
}

\author{
I. E. Umeonyiagu ${ }^{1^{*}}$ and C. C. Nwobi-Okoye ${ }^{2}$

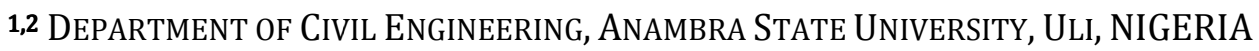 \\ E-mail Addresses: ${ }^{1}$ umeonyiaguikechukwu@yahoo.com,2 chidozien@yahoo.com
}

\begin{abstract}
Cases of collapsed buildings and structures are prevalent in Nigeria. In most of these cases the cause of the collapse could be traced to the strength of the construction materials which is usually concrete. Secondly, experimental determination of the strength of concrete materials used in buildings and structures is quite expensive and time consuming. This work shows the development of a computational model, based on artificial neural networks for the determination of flexural strength of concrete materials made from prevalent coarse aggregate components from Nigeria. The work involves building a multi-layer perception neural network model which uses experimental data obtained from flexural strength test of concrete made from washed gravel. The flexural strength predictions were compared with predictions from an alternative model based on regression analysis. The results of the study show that for the washed gravel based concrete the regression model prediction has a correlation coefficient of 0.92687 and a sum of squares error of 0.51954100 , while the neural network model prediction has a correlation coefficient of 0.98364 and a sum of squares error of 0.00629630 . Generally, the models predicted well, but the neural network model predicted better than the regression model. The result of the study has adequately demonstrated a cheap, simple, very quick and accurate alternative to experimental method of concrete strength determination. The method is also simpler and quicker than analytical methods based on regression analysis. Results obtained suggests an option of immense benefit to civil engineers and construction professionals, which would help in economically determining the strength, as well as economical selection of appropriate mix of construction materials, that is a prelude to building strong and cheap buildings and structures.
\end{abstract}

Keywords: Artificial Neural Network; Concrete; Washed gravel; Regression; Modelling

\section{INTRODUCTION}

Strength being the most important property of concrete determines the quality of concrete. Traditionally, laboratory trial mixes have been used to determine the flexural strengths of concrete. Experimental determination of the strength characteristics of concrete materials is costly and time consuming. Often building contractors in Nigeria in their attempt to cut corners use low quality concrete materials in building constructions leading to cracking and in some cases total collapse of the building or structure. Here in Nigeria cases of collapsed buildings and structures are prevalent, and often lead to massive loss of lives and properties [1, 2]. Olujumoke et al. [1] identified weak concrete mixes as one the major reasons for the collapse of most buildings in Nigeria. This has disastrous socio-economic consequences for the country $[1,2]$.

Building structures with the right materials and proper strength characteristics would eliminate the incidences of collapsed buildings and structures in Nigeria. This will improve the socio-economic wellbeing of the citizens. Finding a potable low cost way of predicting the strength of concrete materials would help in solving the problem of collapsed buildings and structures in Nigeria. One way this could be done is by developing a computational model based on artificial neural network technology for predicting the strength of concrete materials. With mathematical and computational models a designer can easily find the 
best combination of constituent material to balance strength and cost. An artificial neural network (ANN), usually called "neural network" (NN), is a mathematical model or computational model that is inspired by the structure and/or functional aspects of biological neural networks [3]. Concrete for building structures is made with four major constituent materials namely: Portland cement, water, fine and coarse aggregates. But these constituent materials have a range of characteristic parameters such that their combination into a concrete material invariably results in a range of concrete strength. Thus, there is the need to obtain the resulting compressive strength ultimate of the concrete. The strength of concrete in a building or structure is thus varied within the member. However, the structure must be safe enough to resist the applied loads; hence the need to estimate the developed, achieved or resulting concrete strength always as the construction progresses in order to justify investment and safety of lives and property.

\section{EARLIER WORK ON NEURAL NETWORKS IN CONSTRUCTION}

The concept of artificial neurons was first introduced in 1943 [4]. Russell and Norvig [3] stated that since then, much more detailed realistic models have been developed both for neurons and larger systems in the brain leading to the modern field of computational neuroscience. Since the work of McCullooch and Pitts [4], ANN has had wide application in many spheres of life. According to Maier and Dandy [5], in recent years, Artificial Neural Networks (ANNs) have become extremely popular for prediction and forecasting in a number of areas, including finance, power generation, medicine, water resources and environmental science. The utility of artificial neural network models lies in the fact that they can be used to infer a function from observations. This is particularly useful in applications where the complexity of the data or task makes the design of such a function by hand not practical [3].

The tasks to which artificial neural networks are applied tend to fall within the following broad categories:

i. Function approximation, or regression analysis, including time series prediction, fitness approximation and modelling.

ii. Classification, including pattern and sequence recognition, novelty detection and sequential decision making.

Nigerian Journal of Technology, iii. Data processing, including filtering, clustering, blind source separation and compression.

iv. Robotics, including directing manipulators, Computer numerical control.

Much work has been done on the application of ANNs to the prediction of strength of engineering materials. Mukharjee and Biswas [6] in their work applied artificial neural networks to the prediction of the mechanical behaviour of concrete materials at high temperature. Their results were very encouraging which concluded that ANN performed better than existing analytical methods. Oretal and Kawashima [7] in their paper proposed an artificial neural network (ANN) based model, to predict the confined flexural strength and corresponding strain of circular concrete columns. Their study shows the importance of validating the ANN models in simulating physical processes especially when data are limited. The ANN model they developed was also compared to some analytical models and was found to perform reasonably well. Other papers on the prediction of concrete strength using neural networks include: Lee [8], Kasperkiewicz et al. [9], Ahmet et al. [10], Topcu and Saridemir [11] etc.

Maier and Dandy [5] reviewed 43 papers dealing with the use of neural network models for the prediction and forecasting of water resources variables in terms of the modelling process adopted. They identified inadequate model building as the obstacle militating against accurate predictions using artificial networks. They suggested that ANN models must be properly evaluated before its application in time series analysis. Their assertion is corroborated by Chatfield [12] when commenting on the suitability of ANNs for time series analysis and forecasting, who commented thus: "when the dust has settled, it is usually found that the new technique is neither a miraculous cure-all nor a complete disaster, but rather an addition to the analyst's toolkit which works well in some situations and not in others".

It is important to note that a neural network modelling is purely a computational technique. Hence, if one wants to explain an underlying process or mathematical framework that produces the relationships between the dependent and independent variables, it would be better to use a more traditional statistical model like regression analysis. However, if model interpretability is not important, one can often obtain good model results more quickly using a neural network.

Vol. 34, No. 1, January 2015 
Properties of materials used in construction vary from region to region and from country to country. Hence, accordingly, the properties of building materials used in Nigeria are unique and differ significantly with what is obtained in other countries. Here we examined one major coarse aggregate component of concrete used as construction material in eastern Nigeria. This coarse aggregate is washed local gravel.

Concrete is a four component mix of water, cement, fine aggregate and coarse aggregate, of which the important properties are strength (compressive and flexural), deformation under load, durability, permeability and shrinkage. But strength, being considered the most important of these properties determines the quality of the concrete.

The neural network approach is used to predict the flexural strength of concrete materials produced from this coarse aggregate component (washed local gravel). Flexural strength prediction of concrete is necessary in structural design of buildings and structures [1,2]. The neural network model developed has intuitive and theoretical appeal. It was developed based on the assumption that the experimental results were generated by a stochastic process. The model developed was in very good agreement with values obtained from experiment and the theoretical model based on Scheffe's $(4,2)$ regression equations $[13,14]$.

\section{MATERIALS AND METHODS}

\subsection{Experimental Technique and Regression Methodology}

The materials for the mixing and production of concrete were obtained, prepared, and the concrete produced tested. The test results were used to determine the coefficients of the regression model. The material preparation and testing procedure, as well as the regression model development are hereby presented in the following sections.

\subsubsection{Preparation, Curing and Testing of Cube Samples}

The aggregates were sampled in accordance with the methods prescribed in British Standards Institution (BS 882: Part 1: 1992) [15]. The test sieves were selected according to British Standards Institution (BS 410: Part 1: 1986) [16]. The water absorption, apparent specific gravity and bulk density of the coarse aggregates were determined following procedures prescribed in (BS 812: Part 2: 1975) [17]. The Los Angeles abrasion test was carried out in accordance with American Society for Testing and Materials (ASTM Standard C131:1976) [18]. The sieve analysis of the fine and coarse aggregate samples was done in accordance with British Standard Institution (BS 812: Part 1: 1975) [19] and which also satisfied British Standard Institution (BS 882:1992) [20]. The sieving was performed by a sieve shaker. The water used in preparing the experimental samples satisfied the conditions prescribed in British Standard Institution (BS 3148: 1980) [21]. These specimens were cured for 28 days in accordance with British Standard Institution (BS 1881: Part 111: 1983) [22]. The testing was done in accordance with British Standard Institution (BS 1881: Part 116: 1983) [23] using flexural testing machine.

\subsubsection{Regression Model Development Methodology}

The experimental results were fitted to a polynomial regression model based on Scheffe's $(4,2)$ regression model $[13,14]$. The regression model is:

For washed gravel:

$\hat{Y}=3.4 x_{1}+3.5 x_{2}+2.7 x_{3}+2.51 x_{4}+0.14 x_{1} x_{2}-$

$0.1 x_{1} x_{3}-0.1 x_{1} x_{4}-0.25 x_{2} x_{4}+0.94 x_{3} x_{4}$

The regression model assumed that each of the components of concrete ${ }_{2}$ namely: water, cement, fine aggregate and coarse aggregate could be zero or one. But in reality none of these components could be zero or one. Hence, an appropriate transformation of the actual components $\mathrm{z}_{1}, \mathrm{z}_{2}, \mathrm{z}_{3}$ and $\mathrm{z}_{4}$ was used to determine the pseudo components $\mathrm{x}_{1}, \mathrm{x}_{2}, \mathrm{x}_{3}$ and $\mathrm{x}_{4}$ that was used in the regression equations above $[13,14]$.

\subsection{Neural Networks}

As has been previously mentioned, the origin of artificial neurons (ANNs) is based on the work of McCulloch and Pitts [4]. Artificial neurons are building blocks for artificial neural networks. We shall discuss here the structure of artificial neurons and neural network used herein.

\subsubsection{Artificial Neurons}

Artificial neural networks make use of artificial neurons. Artificial neural networks (ANNs) simulate the manner of operation of natural neurons in the human body. The basic unit of operation of an ANN is the neuron shown in Figure 1.

In a typical neuron shown in Figure 1, the input to the neuron $\mathrm{x}_{\mathrm{i}}$ is multiplied by a weighting function $\mathrm{W}_{\mathrm{i}}$ to generate the transformed input $\mathrm{W}_{\mathrm{i}} \mathrm{X}_{\mathrm{i}}$. The transformed inputs are summed to obtain the summed input. The summed input constitutes the variables to the activation/transfer function, g, which generates the output $\mathrm{a}_{\mathrm{j}}$. The output of the transfer function is compared to a threshold value. If the output is greater 
than the threshold value, the neuron is activated and signal is transferred to the neuron output, alternatively, if it is less the signal is blocked.

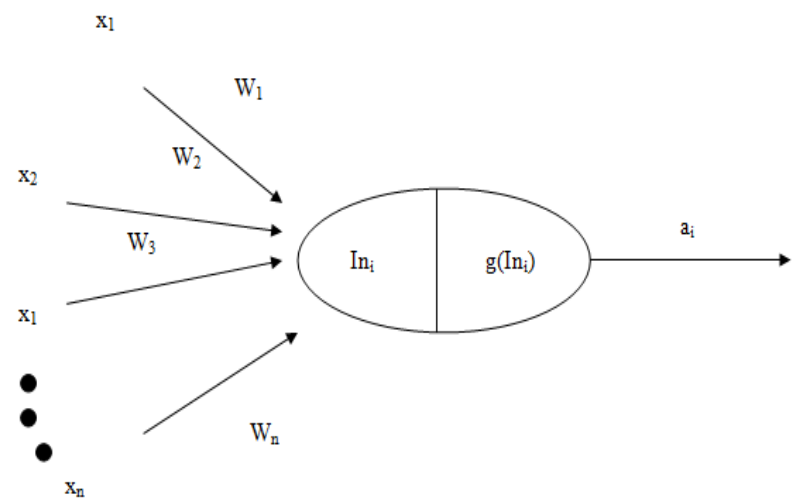

Figure 1: An artificial neuron

Given an input vector $X=x_{1}, x_{2}, \ldots x_{n}$, the activations of the input units are set to $\left(a_{1}, a_{2}, \ldots, a_{n}\right)=\left(x_{1}, x_{2}, \ldots, x_{n}\right)$ and the network computes to:

$\operatorname{In}_{i}=\sum_{j=1}^{n} W_{j, i} a_{j}$
$a_{i}=g\left(\operatorname{In}_{i}\right)$

The transfer function could be a threshold transfer function, a sin function, a sigmoid function, hyperbolic tangent function ${ }_{2}$ etc. Differentiable transfer functions are preferred. Similarly, non linear transfer functions perform better than linear transfer function. Bearing these in mind, in this particular application we chose the sigmoid function. The sigmoid activation function which is given by the equation:

$$
a_{i}=g\left(\operatorname{In}_{i}\right)=\frac{1}{1-e^{-I n_{i}}}
$$

Training the network (learning) could be supervised or unsupervised training. In supervised training, the network is provided with the inputs and appropriate outputs; hence the network is trained with a set of examples in a specified manner. In unsupervised/ adaptive learning, the network is provided with inputs but not the outputs. In this present application, we used the supervised learning, hence, the appropriate network architecture is the feed_forward architecture.

\subsubsection{The Feedforward Network Architecture}

As has been mentioned, the developed neural network models are feed forward multiplayer perceptron networks (MLP). The hidden units as previously noted use the sigmoid activation function. The network model is shown in Figure 2.

In the feed forward network shown in Figure 2, the output of the network is compared with the desired output. The difference between the output and the desired output is known as the error, E. ANNs learn by trying to minimize this error. The learning process uses optimisation algorithms such as LevenbergMarquardt algorithm, gradient descent algorithm, genetic algorithm or other natural optimisation algorithms [3, 24].

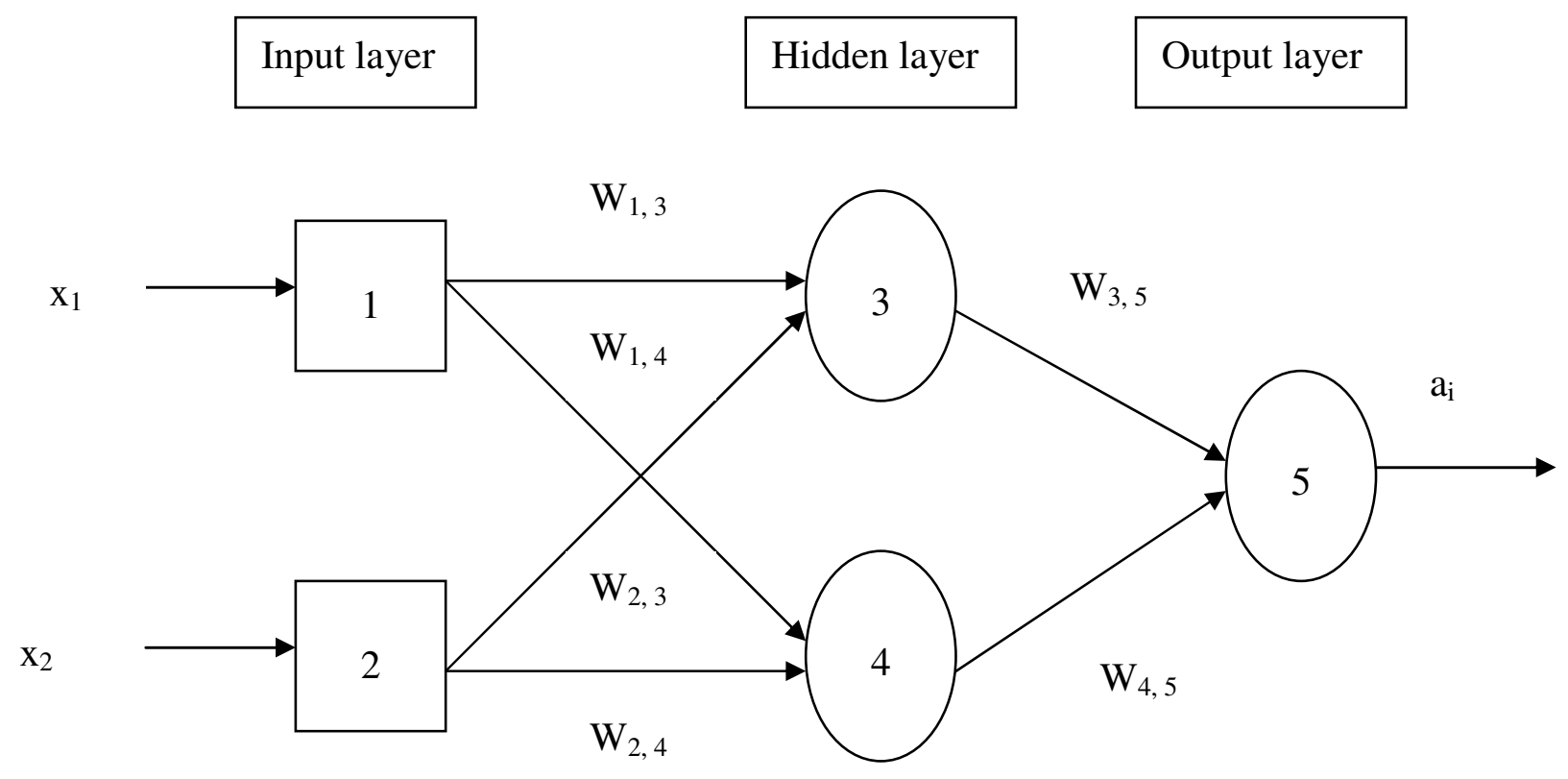

Figure 2: Two-input feed forward neural network model 
These algorithms work by adjusting the weights, $\mathrm{W}_{\mathrm{i}}$, such that the error, E, is minimized. Hence, the learning process uses the sum of squares error criterion $E$ to measure the effectiveness of the learning algorithm [3].

$$
E=\frac{1}{2} E r r^{2} \equiv \frac{1}{2}\left(y-h_{w}(x)\right)^{2}
$$

Here

$\mathrm{y}=\mathrm{Y}=$ the true/experimental value

$$
\hat{Y}=h_{w}(x)
$$

$h_{W}(x)$ is the output of the perceptron.

\section{ANALYSIS, RESULTS AND DISCUSSION}

\subsection{The ANN for Predicting Flexural Strength of Concrete}

Recall that our application is for concrete strength prediction, and we used supervised learning. Hence, Seventy (70\%) percent of the data was used for training, while thirty (30\%) percent was used for testing and validation. The number of epoch was set to 1000.

The epoch was set to 1000 not for any theoretical reasons but to ensure that there is sufficient number of iterations during the learning process. Also learning was fast at this level and the optimum performance was obtained in all cases when the epoch was less than 50. The ANN training was done using LevenbergMarquardt algorithm which performed better than others. Single network architecture was used in the study. The network architecture consists of four input units, two hidden layers with four hidden units (nodes) and one output unit. This structure performed better than other configurations that we tested. The network structure is shown in Figure 4. The inputs $Z_{1}$, $\mathrm{Z}_{2}, \mathrm{Z}_{3}$ and $\mathrm{Z}_{4}$ to the neural network consists of water/cement ratio, cement, fine aggregate and coarse aggregate respectively.

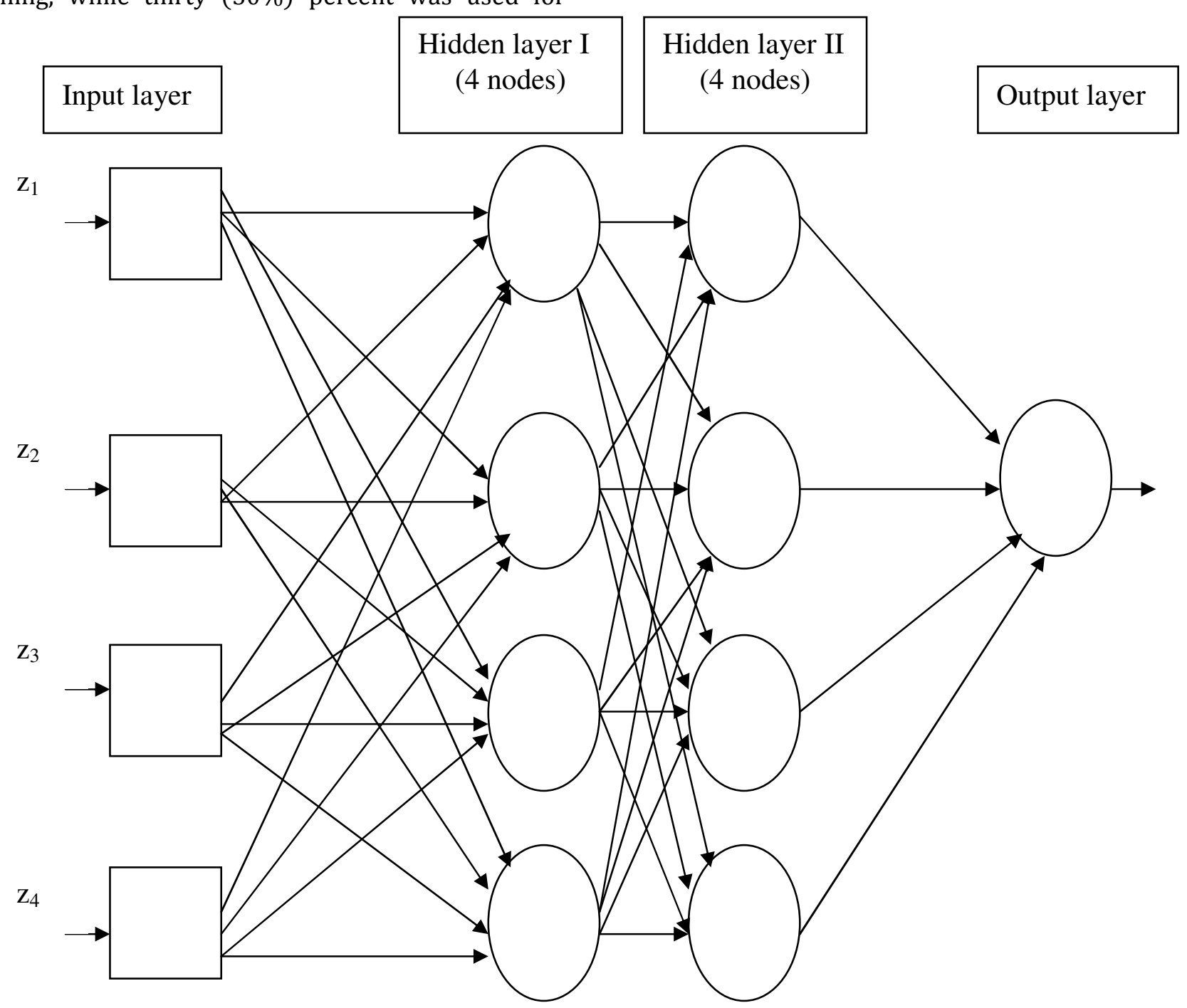

Figure 4: The Four-input, 4 layers feed foreword neural network model 


\subsection{Results}

Table 1 shows the experimentally determined strength, $\mathrm{Y}$, for various mix ratios for river gravel mixtures represented by $\mathrm{Z}$.

Table 1: Results of flexural strengths obtained experimentally

\begin{tabular}{cccccc}
\hline Sample No & $\mathrm{Y}$ & $\mathrm{Z}_{1}$ & $\mathrm{Z}_{2}$ & $\mathrm{Z}_{3}$ & $\mathrm{Z}_{4}$ \\
\hline 1 & 3.4 & 0.6 & 1 & 1.5 & 2 \\
2 & 3.51 & 0.5 & 1 & 1 & 2 \\
3 & 2.7 & 0.55 & 1 & 2 & 5 \\
4 & 2.15 & 0.65 & 1 & 3 & 6 \\
5 & 3.49 & 0.55 & 1 & 1.25 & 2 \\
6 & 3.05 & 0.575 & 1 & 1.75 & 3.5 \\
7 & 2.75 & 0.625 & 1 & 2.25 & 4 \\
8 & 3.08 & 0.525 & 1 & 1.5 & 3.5 \\
9 & 2.77 & 0.575 & 1 & 2 & 4 \\
10 & 2.66 & 0.6 & 1 & 2.5 & 5.5 \\
11 & 3.34 & 0.5625 & 1 & 1.5 & 2.75 \\
12 & 2.86 & 0.6 & 1 & 2 & 3.75 \\
13 & 3.07 & 0.55 & 1 & 1.75 & 3.75 \\
14 & 3.04 & 0.575 & 1 & 1.875 & 3.75 \\
15 & 3.44 & 0.575 & 1 & 1.375 & 2 \\
16 & 3.09 & 0.5875 & 1 & 1.625 & 2.75 \\
17 & 3.06 & 0.6125 & 1 & 1.875 & 3 \\
18 & 3.5 & 0.5125 & 1 & 1.25 & 2.75 \\
19 & 3.1 & 0.5375 & 1 & 1.5 & 3 \\
20 & 2.69 & 0.585 & 1 & 2.25 & 5.25 \\
\hline
\end{tabular}

\subsubsection{Physical and Mechanical Properties of Aggregates}

Sieve analyses of both the fine and coarse aggregates were performed and the grading curves are shown in Figures 5 and 6 . These grading curves showed the particle size distribution of the aggregates. The physical and mechanical properties are summarized in Table 2.

Table 2: Physical and mechanical properties of the river gravel

\begin{tabular}{ll}
\hline \multicolumn{1}{c}{ Properties } & Local Gravel \\
\hline Water absorption & $4.55 \%$ \\
Moisture content & $53.25 \%$ \\
Apparent specific gravity & 1.88 \\
Los Angeles abrasion & $60 \%$ \\
Impact resistance & $22.9 \%$ \\
Bulk density & $1302.7 \mathrm{Kg} / \mathrm{m}^{3}$ \\
Fineness modulus & 70.05 \\
\hline
\end{tabular}

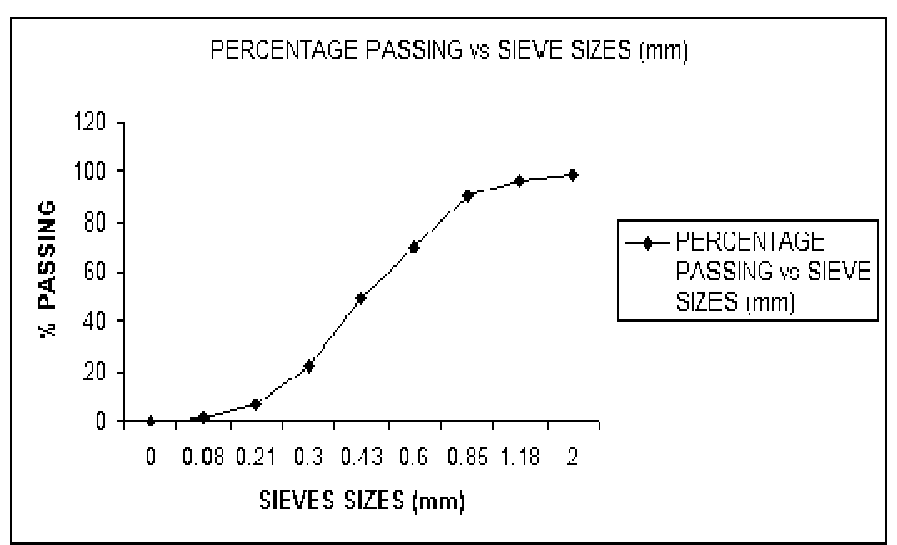

Figure 5: Grading curve for the fine aggregate

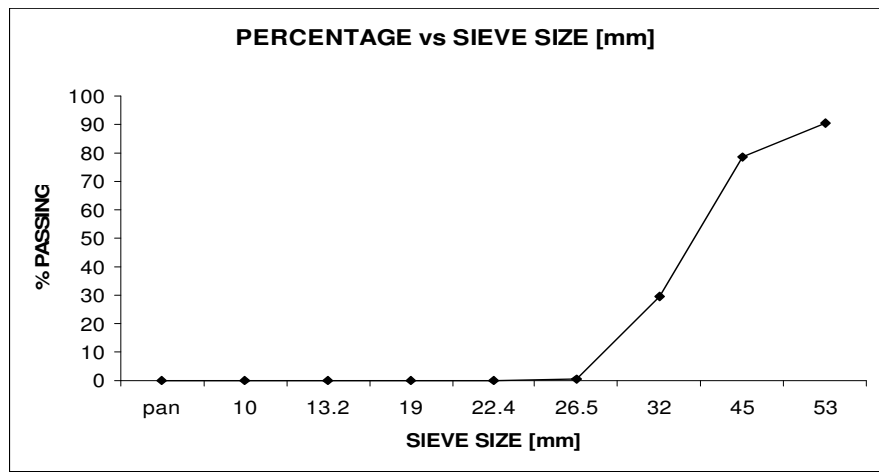

Figure 6: Grading curve for the river gravel

\subsection{ANN Prediction Results}

The results of the experimentally determined concrete strength, analytically determined strength using the regression model, and the strength prediction using neural network models are presented in this section. As Table 1 indicated, the strengths (responses) of the river gravel concrete were a function of the proportions of its ingredients: water, cement, fine aggregate, and coarse aggregates. As shown in Figure 7, correlation coefficient of ANN predictions is 0.98364. The correlation coefficient of regression model predictions is 0.92687 as shown in Table 3. The experimental values were in very good agreement with theoretical values obtained from the Scheffes's regression model and the neural network model. Table 3 shows the comparison of the regression and neural network models.

Table 3: Comparison of ANN and regression models

\begin{tabular}{lll}
\hline Description & $\begin{array}{l}\text { Sum of Squares } \\
\text { Error }\end{array}$ & $\begin{array}{l}\text { Correlation } \\
\text { Coefficient R }\end{array}$ \\
\hline $\begin{array}{l}\text { Regression } \\
\begin{array}{l}\text { Model } \\
\text { Neural network } \\
\text { model }\end{array}\end{array}$ & 0.51954100 & 0.92687 \\
\hline
\end{tabular}




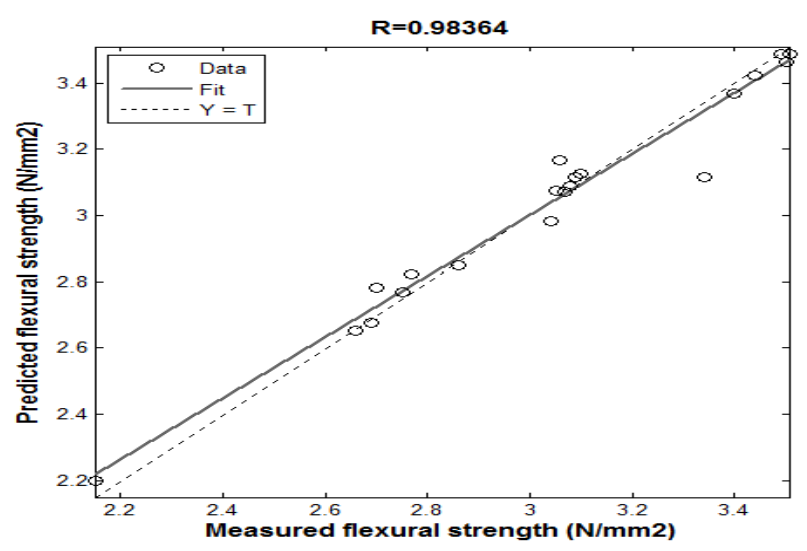

Figure 7: Comparison of ANN predictions with experimental results (river gravel)

\subsection{Discussion}

From the analysis in this work we have seen that the strength of concrete materials depends on the proportion of its ingredients: water, cement, fine aggregate; and coarse aggregates. Figure 7 shows the plot of the artificial neural network predictions for the washed gravel concrete. Generally, the predictions are in good agreement with the experimentally determined flexural strength. Table 2 shows that for the concrete made with river gravel, the regression model prediction has a sum of squares error of 0.51954100 and a correlation coefficient of 0.92687 . Similarly, for the washed gravel based concrete the neural network model prediction has a sum of squares error of 0.00629630 and a correlation coefficient of 0.98364 .

Generally, the neural network models predicted better than the regression models. According to Mukherjee and Biswas [6], guidelines on the configuration of ANNs are not well established. Therefore a trial and error approach is adopted in the selection of network size, training examples and test problems [6]. Past experience plays an important role for selection of the various attributes of the network [6]. Hence, various network configurations were tested before settling for the most appropriate configuration. The variations in the sum of squares error and relative error of the neural network depends on the design architecture [5, $6,25]$.

Generally, the neural network models were bereft of the messy mathematics and statistical analysis required in building the regression model, while at the same time giving good model predictions; hence, would be preferable when the underlying mathematical structure behind the model predictions is irrelevant to the modeler/analyst, and model building is required quickly.

Nigerian Journal of Technology,
Concrete flexural strength determination is very important in civil engineering and in the construction industry [1,7]. It is obvious that neural network models will help in the efficient and accurate determination of concrete strength for building and construction purposes using local materials obtained from Nigeria.

\section{CONCLUSION}

The construction industry is a major component of the economy of any nation. Buildings and structures are indispensable in any modern society. Concrete is the primary building material in Nigeria. As had been noted by Olajumoke et al. [1] and Arum [2], cases of collapsed buildings and structures is endemic in Nigeria. These have resulted in the loss of lives and properties. In addition to these, the economy is impacted negatively $[1,2]$. Often poor concrete mixtures and inadequate knowledge of the role of concrete mixture properties to its strength are to blame $[1,26,27,28]$.

Computational models using neural networks offer a very promising solution to the problem of concrete strength prediction. As we have demonstrated in this application, artificial neural network method for the prediction of flexural strength of concrete from local materials in Nigeria compares favourably with an equivalent mathematical model based on regression analysis. Computational models are simple because it does not involve complex mathematical analysis. Hence, what the engineer needs is good and reliable computer software and a matching hardware to do his analysis. The ubiquity of various computing platforms ranging from desktop PCs, laptops, palmtops, tablets etc means that such analysis is made even easier. The present application was done using a computer laptop which ran the artificial neural network software.

If the recommendations of this work are implemented by adopting the concrete strength prediction aid and making sure construction engineers and technicians stick to it, there will be resultant reduction in cases of collapsed buildings and structures in Nigeria. This will have a very positive effect in terms of growth on the socio-economic condition of the country. The same applies to other countries that are in similar situations as Nigeria.

Finally, neural network models for other common materials used in construction in Nigeria should be developed by engineers and scientists. This will further boost the quality of construction of buildings and other structures. 


\section{Nomenclature, Symbols and Notations}

$\mathrm{z}_{1}=$ water/cement ratio

$\mathrm{z}_{2}=$ water

$\mathrm{z}_{3}=$ fine aggregate

$\mathrm{z}_{4}=$ coarse aggregate

$\mathrm{X}_{1}=$ fine aggregate

$\mathrm{X}_{2}=$ coarse aggregate

$\mathrm{X}_{3}=$ cement

$\mathrm{X}_{4}=$ water

$Y=$ experimentally determined strength

$\hat{Y}=$ network prediction

$\mathrm{W}=$ neural network input weight

$\mathrm{g}=$ network activation function

$a_{\mathrm{i}}=$ neural network input activations

$h_{W}=$ network weighting function

$\mathrm{t}=$ time

\section{REFERENCES}

1. Olajumoke, A.M., Oke, I.A., Fajobi, A.B. and Ogedengbe, M.O. Engineering Failure Analysis of a Failed Building in Osun State, Nigeria. Journal of Failure Analysis and Prevention, 9(1), 2009, pp8-15.

2. Arum C. Verification of Properties of Concrete Reinforcement Bars: Nigeria as a Case Study. Indoor and Built Environment, 17(4), 2008, pp370-376.

3. Russell, S.J. and Norvig, P. Artificial Intelligence: a modern approach. Pearson Educational Inc., Upper Saddle River, New Jersey, USA, 2003.

4. McCulloch, W.S. and Pitts, W. A logical calculus of the ideas imminent in nervous activity, Bulletin and Mathematical Biophysics, 5, 1943, pp 115-133.

5. Maier, H. R. and Dandy G. C. Neural networks for the prediction and forecasting of water resources variables: a review of modelling issues and applications. Environmental Modelling \& Software, 15, 2000, pp 101-124.

6. Mukherjee, A. and Biswas, S.N. Artificial neural networks in prediction of mechanical behavior of concrete at high temperature. Nuclear Engineering and Design, 178(1), 1997, pp1-11.

7. Oretal, A.W.C. and Kawashima, K. Neural Network Modeling of Confined Compressive Strength and Strain of Circular Concrete Columns. Journal of Structural Engineering, 129(4), 2003, pp 554-561.

8. Lee, S.C. Prediction of concrete strength using artificial neural network. Eng. Structures, 25(3), 2003, pp849857.

9. Kasperkiewicz, J., Racz, J. and Dubrawski, A. HPC strength prediction using artificial neural network. J. Comput. Civ. Eng., 9(4), 1995, pp2799-284.
10. Ahmet, O., Murat, P., Erdogan, O., Erdogan, K., Naci, C. and Bhatti, M.A. Predicting the compressive strength and slump high strength concrete using artificial neural network. Constructions and Building Materials, 20(9), 2006, pp769-775.

11. Topcu, I. and Saridemir, M. Predicting of compressive strength of concrete containing y ash using artificial neural networks and fuzzy logic. Computational Material Science, 41(3), 2008, pp305-311.

12. Chatfield, C. Neural networks: Forecasting breakthrough or just a passing fad? International Journal of Forecasting, 9, 1993, pp 1-3.

13. Scheffe, H. Experiment with Mixtures. Royal Statistical Society Journal, Series B, 20, 1958, pp340360.

14. Umeonyiagu, I.E. Mathematical models for the prediction of the concrete strength characteristics of concrete with coarse aggregates of variable sources. Un-published PhD Thesis, Nnamdi Azikiwe University, A wka, Nigeria,

15. "BS 882. Sampling, shape, size and classification. Methods for sampling and testing of mineral aggregates, sands and fillers", British Standard Institution, London, 1992.

16. "BS 410. Specification for test sieves", British Standard Institution, London, 1986.

17. "BS 812, Part 1: Sampling, shape, size and classification. Methods for sampling and testing of mineral aggregates, sands and fillers.Physical properties", British Standard Institution, London, 1975.

18. "ASTM Standard C 131. Tests For Resistance to Abrasion of Small Size Coarse Aggregate by Use of the Los Angeles Machine", American Society for Testing and Materials, 1976.

19. "BS 812, Part 2: Methods for sampling and testing of mineral aggregates, sands and fillers", Physical properties. British Standard Institution, London, 1975.

20. "BS 882. Specification for aggregates from natural sources for concrete", British Standard Institution, London, 1992.

21. "BS 3148. Tests for water for making concrete", British Standard Institution, London, 1980.

22. "BS 1881, Part 111. Method for normal curing of specimen (20 OC)", British Standard Institution, London, 1983.

23. "BS 1881, Part 116. Methods for Determination of Compressive Strength of Concrete Cubes", British Standard Institution, London, 1983.

24. Haupt R.L. and Haupt, S.E. "Practical Genetic Algorithms", John Wiley \& Sons, Inc., Hoboken, New Jersey, USA, 2004. 
25. Atuanya, C.U., Government, M.R., Nwobi-Okoye, C.C., Onukwuli, O.D. Predicting the Mechanical Properties of Date Palm Wood Fibre-Recycled Low Density Polyethylene Composite Using Artificial Neural Network, International Journal of Mechanical and Materials Engineering, 7(1), 2014, pp1-20.

26. Umeonyiagu, I.E. and Nwobi-Okoye, C.C. Predicting the Compressive Strength of Concretes Made with Washed Gravel from Eastern Nigeria Using Artificial Neural Networks. Journal of the Nigerian Association of Mathematical Physics, 23, 2013, pp559-558.
27. Nwobi-Okoye, C.C. and Umeonyiagu, I.E. Predicting the Compressive Strength of Concretes Made with Unwashed Gravel from Eastern Nigeria Using Artificial Neural Networks. Nigerian Journal of Technological Research, 8(2), 2013, pp22-29.

28. Nwobi-Okoye, C.C., Umeonyiagu, I.E. and Nwankwo, C.G. Predicting the Compressive Strength of Concretes Made with Granite from Eastern Nigeria Using Artificial Neural Networks. Nigerian Journal of Technology 32 (1), 2013, pp13-21. 